\title{
Aparición de Melanina como Pigmento Protector en el Encéfalo de Xenopus laevis para Protegerlo de los Efectos de la Radiación Ultravioleta
}

\author{
Emerging Melanin Protective Pigment in the Brain of Xenopus laevis \\ to Protect from the Effects of UV Radiation
}

\author{
H. Díaz Murillo* \& C. Pedemonte Campos***
}

DÍAZ, M. H. \& C. PEDEMONTE, C. C. Aparición de melanina como pigmento protector en el encéfalo de Xenopus laevis para protegerlo de los efectos de la radiación ultravioleta. Int. J. Morphol., 31(3):1120-1123, 2013.

RESUMEN: Con el fin de proteger al organismo de condiciones estresantes, tales como cambios de osmolaridad y de temperatura, además de actuar como pantalla protectora en contra de los rayos ultravioleta (UV). Se ha observado que ciertos anfibios han desarrollado pigmentación en su encéfalo como una posible protección ante el aumento de la radiación UV, causada por el daño en la capa de ozono, la cual estaría alterando al ecosistema. En este trabajo se describe la presencia de pigmentación en el encèfalo de X. laevis durante el desarrollo larvario y su posible función protectora frente a la radiación UV. Para ello, se recolectaron individuos de diferentes estados larvarios, los que fueron obtenidos de distintas localidades de la región de Valparaíso (V región, Chile), para ser procesados con el método corriente H-E y el método de Lillie. En los análisis se pudo evidenciar que la pigmentación correspondía a melanina, que se encontraría en la membrana denominada leptomeninge, la cual recubre al encéfalo y estaría actuando como un filtro protector para evitar daños a nivel del desarrollo en el sistema nervioso de estos anuros. En suma, los rayos UV como agentes deletéreos estarían estimulando la producción de eumelanina en la leptomeninge de estos anfibios, para proteger parte del SNC (encéfalo), como al individuo en sí de posibles alteraciones teratogénicas y/o mutagénicas.

PALABRAS CLAVE: Melanina; Radiación ultravioleta; Xenopus laevis.

\section{INTRODUCCIÓN}

Todo ser vivo que se expone prolongadamente a las ondas lumínicas provenientes del sol, específicamente las ultravioleta (UV), puede sufrir una serie de daños en su organismo ya sean quemaduras, cáncer a la piel, o alteraciones genéticas, entre otros (Instituto de Salud Pública, 2007).

Debido al aumento en el agujero de la capa de ozono, la incidencia de los rayos solares ha aumentado considerablemente afectando a algunos ecosistemas por la acción de la radiación ultravioleta, provocando una disminución de la biomasa de flora y fauna en el planeta (Häder et al., 1998). Sin embargo, se ha observado que en algunos animales terrestres, la exposición directa a estos rayos UV estarían influyendo en procesos de fecundación (Hickman et al., 1998; Paniagua, 1997) y en otros casos en el desarrollo de las crías de peces y anfibios. Y quizás, la desaparición y/o muerte de algunas especies podría estar actuando como un bioindicador del incremento de esta radiación.
Los animales en general han ido generando mecanismos defensivos que les ayudan a mermar los impactos adversos para su desarrollo. Es así que dentro de los pigmentos producidos por los seres vivos, se encuentra la melanina, que es considerada como un agente protector ante la radiación UV, cambios bruscos de temperatura y/u osmolaridad por mencionar algunos. Esta macromolécula se forma a partir de una reacción aeróbica de tipo DOPA por acción de la hormona tirosina y la enzima tirosinasa en el interior de los melanocitos. Además, es insoluble y presenta un elevado peso molecular. Se estima que este pigmento en el encéfalo de anuros podría estar actuando como un filtro protector, reduciendo posibles alteraciones por efectos de la UV (Urán \& Cano, 2008). Un ejemplo de esto es la producción o incorporación depigmentación. Es así que en anfibios de la especie Xenopus laevis en la porción encefálica (SNC), se ha observado el desarrollo inusual de un tipo de pigmento presente en estadios de desarrollo larvario y podría estar actuando como una pantalla protectora. 


\section{MATERIAL Y MÉTODO}

Se recolectó una cantidad de 56 individuos al azar, de la especie Xenopus laevis en diferentes estadios de desarrollo (neurulas, yema caudal, larvas y estadios premetamórficos), los cuales fueron obtenidos de los esteros de las localidades de Belloto Sur, Belloto Centro (332'47" sur y $71^{\circ} 24^{\prime} 26^{\prime \prime}$ oeste respectivamente) y Casablanca ( $33^{\circ} 15^{\prime}$ sur y $\left.17^{\circ} 30^{\prime}\right)$, V región Valparaíso Chile, durante el período estival. Posteriormente, estas muestras fueron procesadas histológicamente y teñidas con el método corriente (H-E) y el método de Lillie y su posterior observación a M.O.

\section{RESULTADOS}

Según los informes meteorológicos que detectaron los índices de radiación ultravioleta obtenido de la Dirección Meteorológica de Chile, comprendidos desde los meses de agosto a noviembre del 2011, indicaron que existían variaciones en la radiación UV, generando cambios graduales, los cuales estarían afectando el medio en el que se desarrollan las crías de los anuros de la especie Xenopus laevis.

La Tabla I muestra el promedio de radiación UV en la atmósfera y la incidencia de esta radiación en el agua. Cada nivel de radiación posee una categoría que varía de insuficiente a dañino (Kinney et al., 2000) y su función es la de indicar las medidas precautorias para resguardarse de la radiación, ya que desde los niveles bajos, la exposición a estos rayos es acumulativa, provocando daño para el organismo. Apoyados en estas variaciones de UV y los datos literarios con los cuales se cuenta se pudo relacionar que el desarrollo del pigmento que se detectó en los encéfalos de los renacuajos tratados con método corriente y posteriormente se empleo el método de Lillie (Lillie, 1954) que permitió corroborar la naturaleza del pigmento.
En la Figura 1 se evidencia un corte transversal de encéfalo de X. laevis en estadio de desarrollo pre-metamórfico, en que se empleó el método corriente (H-E), que permitió observar la distribución de la pigmentación (A) en la membrana leptomeningea (B) que recubre al encéfalo. (C): Región endocondral; (D): Porción diencefálica. En la Figura 2, en que también se observa un corte transversal de encéfalo de X. laevis en el mismo estadio de desarrollo, emplearemos el método histológico de Lillie. Se pudo evidenciar que el pigmento corresponde a melanina (A), la cual se tiñe de un color verde petróleo (según lo descrito por el autor) y se distribuye en la leptptomeninge (B). (C): región endocondral; (D): porción diencefálica. Esta pigmentación se detectó a partir de los estadios de desarrollo 23 al 39 en la membrana que recubre al encéfalo en las regiones de los bulbos olfatorios, lóbulos ópticos (diencéfalo), cerebelo y tronco encefálico.

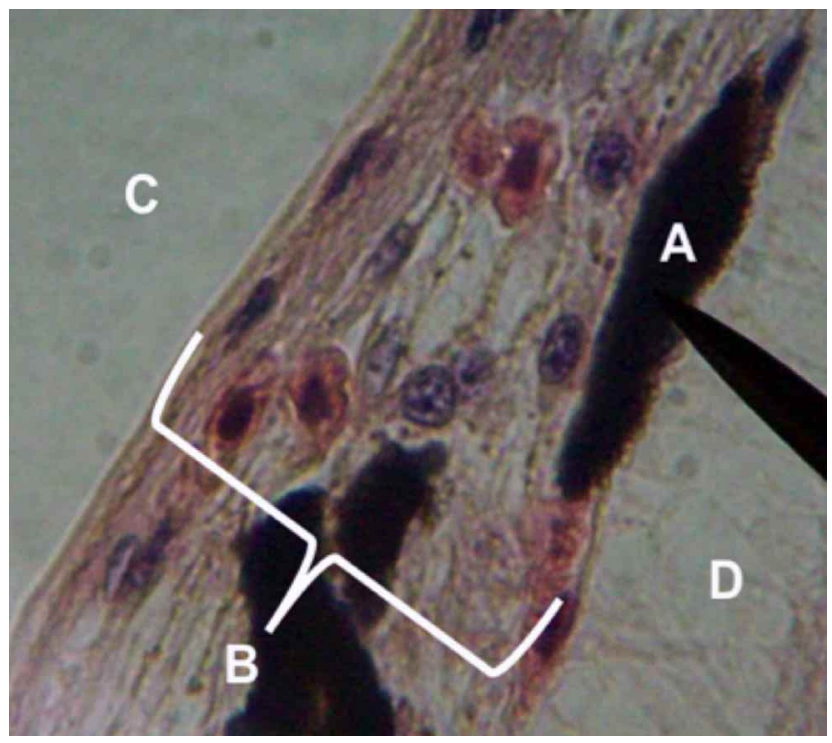

Fig. 1. Corte transversal de encéfalo de Xenopus laevis. Se observa la distribución del pigmento en la leptomeninge del encéfalo de un individuo en estadio 39 de desarrollo.

Leyendas: A = Pigmento (melanina); $\mathrm{B}=$ Región endocondral; $\mathrm{C}$ = Membrana protectora del encéfalo; $\mathrm{D}=$ Porción diencefálica.

Tabla I. Promedio de los niveles del índice de radiación ultravioleta en la atmósfera y la superficie del agua. En los niveles de la radiación ultravioleta bajo, se deben tomar medidas de precaución ya que existe el riesgo de sufrir quemaduras de primer y segundo grado, mientras que en los niveles muy alto se expone al organismo a un cáncer de piel.

\begin{tabular}{lcccc}
\hline & Agosto & Septiembre & Octubre & Noviembre \\
\cline { 2 - 5 } Promedio de * radiación en la atmósfera & 2 & 5 & 7 & 9 \\
Promedio de * radiación en el agua & 3 & 6 & 8 & 10 \\
Nivel de radiación (Kinney et al., 2000) & Bajo & Moderado & Alto & Muy alto \\
\hline
\end{tabular}

* El Índice UV se expresa en una escala numérica lineal abierta cuyo valor más bajo es 0, que indica que la radiación solar no supone ningún riesgo para una persona de salud normal y color de piel medio. 


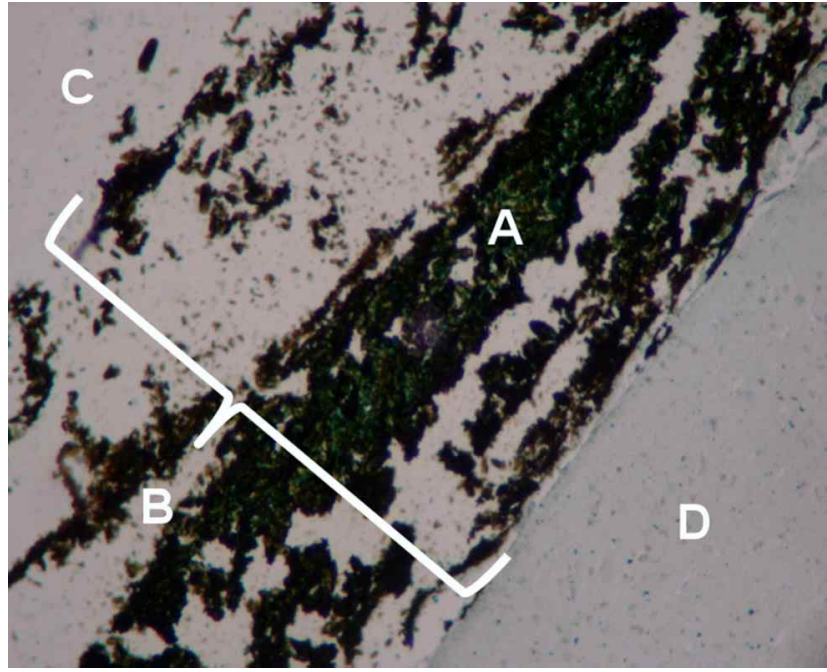

Fig. 2. Corte transversal de encéfalo de Xenopus laevis. Esta figura muestra que el pigmento en la membrana que recubre el encéfalo, corresponde a melanina, ya que esta técnica tiñe la melanina de color verde musgo.

Leyendas: A = Melanina; $\mathrm{B}=$ Región endocondral; $\mathrm{C}=$ Membrana protectora del encéfalo; $\mathrm{D}=$ Porción diencefálica.

\section{DISCUSIÓN}

A través del empleo de la técnica corriente de H-E se dejó en evidencia la presencia de pigmentación en la mem- brana leptomeningea que recubre al encéfalo tanto en los períodos larvarios como a los pre-metamórfico en las porciones de los nervios olfatorios, bulbos olfatorios, lóbulos ópticos (diencéfalo), cerebelo y tronco encefálico. Estos resultados se contraponen a lo postulado por Kordylewski (1984), quien sólo destaca la presencia de pigmentos en pequeños islotes a nivel diencefálico, específicamente en la porción de los lóbulos ópticos, argumentando que dichos pigmentos podrían ser melanina. Sin embargo, en este trabajo se pudo corroborar que la pigmentación correspondía a melanina, ya que se evidenció a través del método histológico de Lillie, que marcó los pigmentos de melanina de un color verde musgo. Por lo tanto, se puede establecer que el pigmento que se evidenció en la membrana protectora del encéfalo de los anuros estudiados era melanina, ubicándose específicamente en la región apical encefálica y posiblemente tendría un rol protector a los rayos UV. Esto concuerda con otros autores (Urán \& Cano), que afirman que la melanina tiene una función de proteger al organismo de los rayos solares. Donde el color oscuro absorbe todo el espectro visible de dichos rayos, cumpliendo una función de pantalla protectora. En resumen, esto podría explicar que estos anfibios protejan su SNC (encéfalo) ya que se encuentran provistos de una piel muy delgada y translúcida, donde la función de la melanina sería proteger al individuo de condiciones ambientales que le puedan ser adversas, como lo es la radiación ultravioleta, además de los posibles efectos que se estarían generando por la contaminación antropogénica.

DÍAZ, M. H. \& C. PEDEMONTE, C. C. Emerging melanin protective pigment in the brain of Xenopus laevis to protect from the effects of UV radiation. Int. J. Morphol., 31(3):1120-1123, 2013.

SUMMARY: It has been observed that certain amphibians have developed pigmentation in brain as a possible increased protection against UV radiation, caused by damage to the ozone layer, which would alter the ecosystem. In this paper we describe the presence of pigment in the brain of $\mathrm{X}$. laevis during larval development and possible protective function against UV radiation. To do this, we collected individuals at various larval stages, which were obtained from different locations in Valparaiso (V Region, Chile ), to be processed with $\mathrm{HE}$ and the method of Lillie. In the analysis it was evident that pigmentation corresponded to melanin, which would be in the membrane called leptomeninges, which covers the brain and would be acting as a protective filter to prevent damage to the level of development in the nervous system of these frogs. In addition, UV rays would be deleterious agents stimulating production of eumelanin in the leptomeninges of these amphibians, to protect the CNS (brain), and the individual itself of potential teratogenic or mutagenic alterations.

KEY WORDS: Melanin; Ultraviolet radiation; Xenopus laevis.

\section{REFERENCIAS BIBLIOGRÁFICAS}

Häder, D. P.; Kumar, H. D.; Smith, R. C. \& Worrest, R. C. Effects on aquatic ecosystems. J. Photochem. Photobiol. B, 46:53-68, 1998.
Hickman, C. P.; Roberts, L. S. \& Larson, A. Principios integrales de zoología. $10^{\mathrm{a}}$ ed. Madrid, Madrid, McGraw-Hill Interamericana, 1998. 
DÍAZ, M. H. \& C. PEDEMONTE, C. C. Aparición de melanina como pigmento protector en el encéfalo de Xenopus laevis para protegerlo de los efectos de la radiación ultravioleta. Int. J. Morphol., 31(3):1120-1123, 2013.

Instituto de Salud Pública. Informe técnico exposición laboral a la radiación ultra violeta de origen. Sección radiaciones ionizantes y no ionizantes sección elementos de protección personal. Departamento de Salud Ocupacional y Contaminación Ambiental. Santiago de Chile, Ministerio de Salud, 2007.

Kinney, J. P.; Long, C. S. \& Geller, A. C. The Ultraviolet Index: A Useful Tool. Dermatol. Online J., 6(1):2, 2000

Kordylewski, L. Egg pigmented is accumulated in the tadpole's brain. Experientia, 40(3):277-9, 1984.

Lillie, R. D. Histopathologic technique and practical histochemistry. New York, McGraw-Hill, 1954.

Paniagua, R. Citología e histología vegetal y animal Biología de las células y tejidos animales y vegetales. Madrid, McGrawHill Interamericana, 1997.

Urán, J. M. E. \& Cano, R. L. E. Melanina: implicaciones en la patogénesis de algunas enfermedades y su capacidad de evadir la respuesta inmune del hospedero. Infect., 12(2):357-77, 2008.

\author{
Dirección para Correspondencia: \\ Dr. Hugo Díaz Murillo \\ Depto. de Biología y Cs. Ambientales \\ Facultad de Ciencias \\ Universidad de Valparaíso \\ Valparaíso \\ CHILE
}

Email: hugo.diaz@uv.cl

Recibido : 13-04-2013

Aceptado: 04-08-2013 